pharmaceutical companies, Antonis Fanouriakis Paid instructor for: Paid instructor for Enorasis, Amgen, Speakers bureau: Paid speaker for Roche, Genesis Pharma, Mylan

DOI: 10.1136/annrheumdis-2020-eular.6255

\section{FRI0171 THE CHANGES OF IMMUNE FUNCTION AND CLINICAL INDEXES WITH SYSTEMIC LUPUS ERYTHEMATOSUS AFTER IMMUNOREGULATORY COMBINATION THERAPIES}

X. Liu' ${ }^{1}$, X. Liü ${ }^{2}, \mathrm{H} . \mathrm{Hou}^{3}, \mathrm{X} . \mathrm{LI}^{1}$ on behalf of Li Xiaofeng Team. ${ }^{1}$ The Second Hospital of Shanxi Medical University, Taiyuan, China; ${ }^{2}$ Taiyuan University of Technology, Taiyuan, China; ${ }^{3}$ Inner Mongolia Normal University, Huhehaote, China

Background: Recent studies have reported that some drugs such as low-dose interleukin-2, rapamycin, metformin, retinoic acid and coenzyme Q10 could promote the proliferation and functional recovery of regulatory T cells (Treg) in patients with autoimmune diseases. However, the effects on the balance of Treg cells and pro-inflammatory lymphocytes and long-term efficacy have rarely been reported.

Objectives: To evaluate the changes of peripheral lymphocyte subsets, conventional drugs and remission rate in patients with systemic lupus erythematosus (SLE) after immunomodulatory combination therapies.

Methods: A total of 189 patients with SLE from the Second Affiliated Hospital of Shanxi Medical University from January 2016 to October 2019 were enrolled, who were divided into well-controlled group and untargeted control group taking a full consideration of the patient's symptoms, signs and related laboratory findings. We measured the absolute counts of $\mathrm{B}, \mathrm{NK}, \mathrm{CD} 8+\mathrm{T}$ and helper T 1 (Th1), helper T 2 (Th2), helper T 17 (Th17) and Treg cells in peripheral blood of patients before immunomodulatory combination therapies and during the 3 months and 6 months of follow-up and 190 sex- and age- matched control individuals using flow cytometry. Moreover, the ratios of various cells to Treg cells were calculated. Results: Compared with healthy controls, Treg cells in SLE patients were significantly lower before the treatment with immunomodulator, while the ratios of various pro-inflammatory lymphocytes to Treg cells (such as Th2/ Treg, Th17/Treg, CD8+T/Treg, etc.) were higher. After 3 months and 6 months with immunomodulatory therapy, the absolute number of Treg cells in peripheral blood of SLE patients increased obviously reaching to normal level. Accordingly, the ratios of various pro-inflammatory lymphocytes to Treg cells recovered. At the same time, the dose of glucocorticoid and disease-modifying antirheumatic drugs (DMARDs) decreased distinctly. Additionally, the well-controlled group was able to maintain a high remission rate, and the untargeted control group could achieve a higher response rate after immunomodulatory treatment.

Conclusion: The imbalance between pro-inflammatory lymphocytes and Treg cells caused by the significant decrease of Treg cells may be the main cause of SLE. And immunomodulatory combination therapies we came up with may reverse the imbalance of proinflammatory lymphocytes and Treg cells, which is an potential and effective treatment for SLE.

References:

[1] Noack M, Miossec P. Th17 and regulatory T cell balance in autoimmune and inflammatory disease[J]. Autoimmun Rev, 2014, 13(6): 668-677.

[2] Yu A, Snowhite I, Vendrame F, et al. Selective IL-2 responsiveness of regulatory $T$ cells through multiple intrinsic mechanisms supports the use of low-dose IL-2 therapy in type 1 diabetes. Diabetes. 2015;64: 2172-2183.

[3] Schuiveling M, Vazirpanah N, Radstake TRDJ, Zimmermann M, Broen JCA. Metformin, A New Era for an Old Drug in the Treatment of Immune Mediated Disease?[J]. Curr Drug Targets, 2017;18:1-15.

Table1. The changes of remission rate in the no-remission group during follow-up.

\begin{tabular}{lllll}
\hline Follow-up period & Total patients & Remission & No-remission & Remission rate(\%) \\
\hline Baseline & 92 & 0 & 92 & 0 \\
3 Months & 72 & 33 & 39 & $45.8^{\mathrm{a}}$ \\
6 Months & 74 & 42 & 32 & $56.8^{\mathrm{a}}$ \\
\hline
\end{tabular}

a: Compared with baseline; b: Compared with 3 months.

Acknowledgments: We would like to express our sincere gratitude to all our coworkers and collaborators, Jing Luo, Xiangcong Zhao, Chen Zhang, Qi Wu, Congcong Liang, and Rui Fu for their technical support.

Disclosure of Interests: None declared

DOI: 10.1136/annrheumdis-2020-eular.5801

\section{\begin{tabular}{|l|l|l|l|}
\hline FRI0172 & THE INFLUENCE OF CALCINEURIN INHIBITORS
\end{tabular} ON DEVELOPMENT OF CANCER IN PATIENTS WITH SYSTEMIC LUPUS ERYTHEMATOSUS: A RETROSPECTIVE OBSERVATIONAL STUDY IN THE LUNA REGISTRY}

K. Ichinose ${ }^{1}$, T. Igawa ${ }^{1}$, M. Okamoto ${ }^{1}$, A. Takatani ${ }^{1}$, N. Yajima ${ }^{2}$, K. E. Sada ${ }^{3}$, R. Yoshimi ${ }^{4}$, Y. Shimojima ${ }^{5}$, S. Ono ${ }^{6}$, H. Kajiyama ${ }^{7}$, S. Sato ${ }^{8}$, M. Fujiwara ${ }^{9}$, A. Kawakami ${ }^{1}{ }^{1}$ Nagasaki University Graduate School of Biomedical Sciences, Department of Immunology and Rheumatology, Division of Advanced Preventive Medical Sciences, Nagasaki, Japan; ${ }^{2}$ Showa University School of Medicine, Division of Rheumatology, Department of Internal Medicine, Shinagawa-ku, Tokyo, Japan; ${ }^{3}$ Okayama University Graduate School of Medicine Dentistry and Pharmaceutical Sciences, Department of Nephrology, Rheumatology, Endocrinology and Metabolism, Okayama, Japan; ${ }^{4}$ Yokohama City University Graduate School of Medicine, Department of Stem Cell and Immune Regulation, Yokohama, Japan; ${ }^{5}$ Shinshu University School of Medicine, Department of Medicine (Neurology and Rheumatology), Matsumoto, Japan: ${ }^{6}$ Yokohama City University Medical Center, Center for Rheumatic Diseases, Yokohama, Japan; ${ }^{7}$ Saitama Medical University, Department of Rheumatology and Applied Immunology Faculty of Medicine, Saitama, Japan; ${ }^{8}$ Fukushima Medical University School of Medicine, Department of Rheumatology, Fukushima, Japan; ${ }^{9}$ Yokohama Rosai Hospital, Department of Rheumatology, Yokohama, Japan

Background: It has been reported that the incidence of cancer in patients with systemic lupus erythematosus (SLE) is higher than that in healthy individuals, but the findings are inconsistent ${ }^{1}$. In the transplantation field, a few studies indicated an association between the use of immunosuppressants and an increased risk of cancer ${ }^{2}$. Calcineurin inhibitors (CNIs), which include cyclosporine and tacrolimus, have been used for $>30$ years to treat renal and extrarenal manifestations of SLE, but the effects of exposure to CNIs among SLE patients have not been established.

Objectives: We investigated the incidence of various cancers (including cervical dysplasia) among SLE patients registered in the LUpus registry of NAtionwide institution (LUNA). We also investigate whether the registrants' exposure to CNIs increased the risk of cancer.

Methods: We calculated the standardized incidence ratio (SIR) of cancer among SLE patients based on the age-standardized incidence rate of cancer reported by Japan's Ministry of Health, Labour and Welfare. A multivariate analysis of the risk of cancer was performed using the covariates of age, smoking history, $\mathrm{CNI}$ treatment history, maximum steroid dose in the past, and Systemic Lupus International Collaboration Clinics/American College of Rheumatology Damage Index [SDI]) value (excluding the occurrence of cancer) at the time of the patient's registration.

Results: We studied 714 patients (663 females; $88.9 \%$ ). The median age at registry was 44 [interquartile range (IQR): 35-56] years. The median past max. steroid dose was $40 \mathrm{mg} /$ day (IQR: $30-60 \mathrm{mg} /$ day), and the SDI at registration was 1 (IQR 0-2). Smoking history was present in 248 patients (34.9\%), and 53 patients (7.4\%) experienced cancer complications. Gynecologic malignancies accounted for $71 \%$ of all cancers, including 12 cervical dysplasia cases. The standardized incidence rate of cancer in these SLE patients was $1.46(95 \% \mathrm{Cl}: 1.07-1.85, \mathrm{p}<0.01)$. The multivariate analysis showed that a $\mathrm{CNI}$ treatment history was not a risk factor for the development of cancer (OR 1.76, $95 \% \mathrm{Cl}: 0.63-4.88, \mathrm{p}=0.30$ ). After the covariance was adjusted for the propensity score, the risk of cancer in the CNIs group was not increased compared to the non-CNIs group (adjusted OR 2.46 95\% Cl: 0.68-8.91, $\mathrm{p}=0.20$ ).

Conclusion: The incidence of cancer in SLE was higher in the LUNA cohor than in the general population. Our results suggest that $\mathrm{CNI}$ treatment for individuals with SLE is not a risk factor for the development of cancer. References:

[1] Ladouceur A. et.al, Expert Rev Clin Immunol. 2018 Oct;14(10):793-802. [2] Gutierrez-Dalmau A. et.al, Drugs 2007;67(8):1167-98.

Disclosure of Interests: None declared

DOI: 10.1136/annrheumdis-2020-eular.1379

FRI0173

IDENTIFICATION OF RISK FACTORS FOR DEVELOPMENT OF OSTEONECROSIS IN SYSTEMIC LUPUS ERYTHEMATOSUS

R. Kallas ${ }^{1}$, J. LI' ${ }^{1}$, M. Petri ${ }^{1} .{ }^{1}$ Johns Hopkins University School of Medicine, Baltimore, United States of America

Background: Patient with Systemic Lupus Erythematosus (SLE), particularly those who received corticosteroids are at a high risk of osteonecrosis (ON). 